\title{
Effet d'une réduction du rayonnement incident sur l'émission des racines adventives du maïs en début de cycle
}

\author{
S Pellerin \\ avec la collaboration technique de A Blatz et B Petit
}

INRA, laboratoire d'agronomie, BP 507, 68021 Colmar, France

(Reçu le 17 août 1989; accepté le 25 octobre 1990)

\begin{abstract}
Résumé - On a étudié au champ l'effet d'une réduction de l'alimention carbonée sur l'émission en début de cycle des racines adventives du maïs (Zea mays $L$ variété Déa). Les plantes ont été ombrées grâce à des filets posés au stade 4-5 feuilles visibles en 1987 et 1988. Trois types de filets ont été utilisés. Le rayonnement mesuré à l'intérieur des ombrages correspondait respectivement à $84 \%$ (traitement I), $30 \%$ (traitement II) et $20 \%$ (traitement III) du rayonnement extérieur. Des mesures de température de l'air et de température du sol ont également été effectuées sous les ombrages. Des plantes ont été prélevées régulièrement pour suivre la croissance des parties aériennes et l'émission des racines adventives. A la récolte, on a compté le nombre final de racines par entre-nœud.

Les résultats montrent qu'il y a eu sous les ombrages une moindre croissance des plantes et un retard de l'apparition des feuilles et des racines adventives. Quel que soit le traitement, on observe une même relation linéaire entre le nombre d'entre-nœuds ayant émis des racines et le nombre de feuilles visibles. La synchronie entre les émissions foliaires et racinaires n'a donc pas été modifiée. On observe également une relation entre la biomasse aérienne des plantes et le nombre total de racines adventives émises. Sur les premiers entre-nœuds $\left(E_{2}-E_{5}\right)$, le nombre final de racines est identique dans les traitements ombrés et non ombrés bien que l'émission racinaire sur ces entre-nœuds ait eu lieu pendant la période d'ombrage. Sur ces entre-nœuds, le nombre final de racines émises ne serait donc pas régulé en fonction de l'alimentation carbonée de la plante. Sur l'entrenœud $6\left(E_{6}\right)$, par contre, le nombre de racines est significativement plus faible dans les traitements ombrés quand l'émission sur cet entre-nœud a eu lieu pendant la période d'ombrage. Ces résultats sont convergents avec les observations d'autres auteurs qui ont montré qu'à partir de $E_{6}$ le nombre de racines émises est inférieur au nombre d'ébauches racinaires présentes.
\end{abstract}

\section{Zea mays = maïs / système racinaire / racine adventive / ombrage / nutrition carbonée}

Summary - Effect of shading on the emission of adventitious roots of maize during the early stages. The effect of carbon availability on the emission of maize adventitious roots was studied by shading plants. Maize was grown outdoors in 1987 and 1988 and plants were shaded with plastic nets over the canopy. Three kinds of shading nets were used. The radiation measured underneath was $84 \%$ (treatment 1), $30 \%$ (treatment II) and $20 \%$ (treatment III) respectively of the radiation measured above the nets (fig 2). The shading nets were spread over the canopy when plants had 4-5 visible leaves. Air and soil temperatures were recorded in the shaded treatments. Plants were sampled regularly for dry matter measurements and adventitous root counting. At harvest the final number of roots per internode was counted. Results showed that plant growth was affected by shading (fig 3). The appearance of leaves and adventitious roots was also delayed in the shaded treatments (fig 4). There was a linear relationship between the number of internodes with emerged roots and the number of visible leaves (fig 5). The synchronism between leaf and root rates of appearance was not affected by shading. A relationship was also observed between total number of emerged roots and aerial biomass (fig 6). On the first internodes (called $E_{2}-E_{5}$ ) the final number of emerged roots was similar in shaded and unshaded treatments although root appearance on these internodes occurred during the shaded period (fig 7). On these internodes, the number of roots does not seem to be regulated by carbon availability. On the following internode (called $E_{6}$ ) the number of roots was lower in shaded treatments when root appearance on this internode occurred during the shaded period. This result is consistent with the observations of other authors who have shown that on upper internodes $\left(E_{6}-E_{8}\right)$ there were fewer emerged roots than root primordia. 


\section{INTRODUCTION}

Plusieurs auteurs ont proposé récemment des modèles tridimensionnels d'architecture racinaire de plantes cultivées (Porter et al, 1986; Pagès et Ariès, 1988; Diggle, 1988; Pagès et al, 1989). Ils permettent de simuler le développement et la croissance du système racinaire dans le volume de sol exploré par initiation, allongement et ramification de petits segments représentant les racines. Un intérêt de ces modèles est qu'ils permettent le calcul de variables de sortie (distances entre racines ou nombre d'extrémités apicales par exemple) pertinentes par rapport aux possibilités d'extraction d'eau ou d'éléments minéraux. Une des difficultés est d'y intégrer les mécanismes de régulation qui conditionnent la mise en place de l'appareil racinaire. Ainsi, dans les modèles existant actuellement, le développement et la croissance des racines ne dépendent pas de la disponibilité en produits de la photosynthèse. On sait pourtant que la fourniture d'assimilats par les parties aériennes conditionne fortement la croissance des racines (Del Castillo et al, 1989). L'application d'un ombrage entraîne une réduction de la biomasse racinaire (Aresta et Fukai, 1984). Sur blé, Vincent et Grégory $(1989 a$, b) ont observé une relation entre la quantité de rayonnement interceptée par la culture et la longueur totale de racines. L'utilisation de ces connaissances dans un modèle d'architecture racinaire est cependant difficile car les variables mesurées sont généralement la masse ou la longueur totale de racines sans distinction de l'identité des racines ou des paramètres qui sont modifiés (nombre de racines émises, vitesse d'allongement, nombre de ramifications, etc). L'écriture d'un modèle d'architecture qui intègre une régulation de la croissance par la disponibilité en produits de la photosynthèse nécessite une identification préalable des caractéristiques du système racinaire qui sont susceptibles d'être affectées.

L'objectif du travail présenté dans cet article est d'étudier, chez le maïs, l'effet d'une réduction de l'alimentation carbonée sur le nombre et le rythme d'apparition des racines adventives en début de cycle. L'existence d'une variabilité intra- et interparcellaire du nombre de racines par entre-nœud avait été mise en évidence (Picard et al, 1985; Jordan et al, 1988). L'origine de cette variabiité n'est pas connue pour l'instant.

\section{MATÉRIEL ET MÉTHODES}

L'essai a été réalisé au champ en 1987 et 1988 sur sol limoneux profond (Domaine expérimental INRA Colmar, Alsace). L'horizon $0-0,30 \mathrm{~m}$ a les caractéristiques suivantes : argile $20,7 \%$; limon fin $31,1 \%$; limon grossier $38,2 \%$; sable fin $5,2 \%$; sable grossier $2,9 \%$; matière organique $1,8 \%$; $\mathrm{pH}$ eau $8,3, \mathrm{P}_{2} \mathrm{O}_{5} \quad 0,25 \%$; $\mathrm{K}_{2} \mathrm{O} 0,21 \%$; $\mathrm{MgO} 0,27 \%$. La préparation du sol et la fertilisation ont été conduites selon les normes locales de la grande culture : labour d'hiver et reprise au vibroculteur juste avant semis; apport de $\mathrm{N}, \mathrm{P}_{2} \mathrm{O}_{5}$ et $\mathrm{K}_{2} \mathrm{O}$ de $140 \mathrm{~kg} / \mathrm{ha}$. La parcelle a été semée en maïs (variété Déa) le 27 avril 1987 et le 5 mai 1988. La densité réelle mesurée au stade 4 feuilles visibles était de 8,1 et 8,3 plantes $\cdot m^{-2}$ respectivement. Les caractéristiques climatiques des 2 années d'étude sont indiquées figure 1. Pendant les 4 semaines qui ont suivi le semis, le climat a été plus froid et plus humide en 1987 qu'en 1988.

Afin de réduire leur alimentation carbonée, des plantes ont été ombrées grâce à des filets reposant sur un support parallélépipédique de $15 \mathrm{~m} \times 3,5 \mathrm{~m}$ de surface au sol ( 5 interrangs) et 1,2 $\mathrm{m}$ de haut. Le filet couvrait le dessus et les 4 côtés du parallélépipède. Sur les côtés, un espace de $0,15 \mathrm{~m}$ a été ménagé entre la base du filet et le sol de façon à assurer une meilleure circulation de l'air entre l'extérieur et l'intérieur. Trois types de filets ont été utilisés de façon à faire varier l'intensité de l'ombrage. Le rapport des surfaces claires et obscures a été déterminé sur analyseur d'image (Quantel, Intellect 100). La trame obscure correspondait respectivement à $15,6 \%, 58,8 \%$ et $71,9 \%$ de la surface totale pour les filets notés respectivement I, II et III. Les filets ont été mis en place le 26 mai 1987 et le 27 mai 1988 , aux stades 4,0 et 5,4 feuilles visibles, c'est-à-dire peu après le début de la période d'autotrophie (Bourdu et Grégory, 1983). Ils ont été retirés le 22 juillet 1987 et le 7 juillet 1988 . En 1987 , seuls les filets II et III ont été mis en place. On a effectué des mesures de rayonnement au-dessus du couvert dans les différents traitements par journée en-

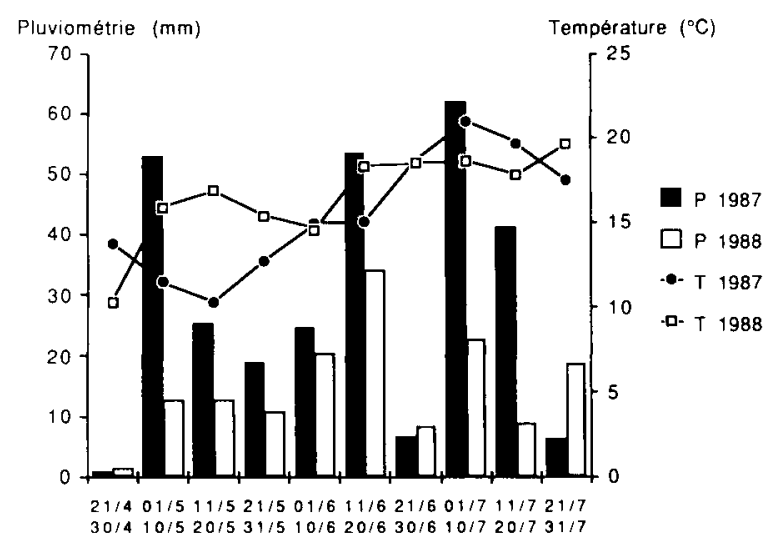

Fig 1. Pluviométrie et température moyennes décadaires en 1987 et 1988 à Colmar. 
soleillée le 24 juin 1988. Des mesures de photosynthèse sur feuilles ont été réalisées à l'aide d'un appareil de type Licor-Li 6000 . Les températures minimales et maximales de l'air ont été mesurées dans les différents traitements en 1987. En 1988, on a mesuré les températures minimales et maximales du sol entre le 15 juin et le 6 juillet.

Des prélèvements de plantes ont été effectués régulièrement de façon à suivre la croissance des parties aériennes et l'apparition des racines adventives. Sous les ombrages, seuls les 3 rangs du milieu ont été utilisés en laissant sur chaque rang des pieds de bordure entre les prélèvements successifs. Sur chaque plante prélevée, on a noté le nombre de feuilles visibles et étalées, la biomasse aérienne et le nombre de racines émises par entre-nœud. A la récolte, on a effectué un comptage final du nombre de racines par entre-nœud. Ceux-ci ont été numérotés conformément au système de notation proposé par Girardin et al (1986). Le mésocotyle est assimilé par convention à un entre-nœud, noté $E_{1}$, et le premier entre-nœud portant des racines adventives est noté $E_{2}$. Une racine est comptée comme émise dès qu'elle a percé la gaine couvrant l'entre-nœud dont elle est issue.

\section{RÉSULTATS}

\section{Résultats des mesures de température et du rayonnement}

Les températures de l'air mesurées en 1987 sous les ombrages ont été très peu différentes des températures mesurées à l'extérieur dans les mêmes conditions. Elles sont un peu inférieures sous les ombrages aux fortes températures. $T_{0}$, $T_{1 \mid}$ et $T_{\text {III }}$ désignant respectivement les températures moyennes journalières à l'extérieur et sous les ombrages II et III, les régressions linéaires obtenues entre ces variables sont les suivantes:

$$
\begin{array}{ll}
T_{I I}=0,969 T_{0}+0,463, & r^{2}=0,976 ; \\
T_{I I I}=0,945 T_{0}+0,785, & r^{2}=0,979 .
\end{array}
$$

En moyenne, pendant la période où les mesures ont été effectuées, les écarts de température de l'air entre l'extérieur et sous les ombrages II et III ont été de 0,08 et $0,18^{\circ} \mathrm{C}$ respectivement. Dans le cas des températures du sol mesurées en 1988 , les écarts moyens entre traitements pendant la période de mesure sont nettement supérieurs : 3,35 et $3,80^{\circ} \mathrm{C}$ respectivement entre l'extérieur et les ombrages II et III.

Dans la figure 2, sont consignées les mesures de rayonnement effectuées le 24 juin 1988 à plusieurs heures de la journée dans les différents traitements. En moyenne, le rayonnement mesuré correspond à $84,3 \%$ du rayonnement extérieur sous l'ombrage I, $30,0 \%$ sous l'ombrage II et $20,3 \%$ sous l'ombrage III. Ces pourcentages sont un peu plus faibles en début et en fin de journée (respectivement $80,3 \%, 27,6 \%$ et $15,9 \%$ à 7 h 24 , heure solaire par exemple). Pour les ombrages II et III, ces valeurs sont inférieures aux rapports (surface claire/surface totale) mesurés sur les filets (respectivement $41,2 \%$ et $28,1 \%$ ). Cela peut être dû à la géométrie des ombrages et au fait que le rayonnement incident n'est pas perpendiculaire à la surface des filets. La plus forte réduction du rayonnement constatée en début et en fin de journée tend à confirmer cette hypothèse. Les mesures de photosynthèse sur feuilles réalisées le 16 juillet 1987 montrent qu'il y a bien eu, sous les ombrages, réduction de l'alimentation carbonée (respectivement $1,314,0,696$ et $0,480 \mathrm{mg}\left(\mathrm{CO}_{2}\right) \cdot \mathrm{m}^{-2} \cdot \mathrm{s}^{-1}$ à l'extérieur et sous les ombrages II et III.

\section{Développement et croissance des parties aériennes}

La figure $3 a, b$ représente l'évolution de la biomasse aérienne par plante dans les différents traitements pendant la période ombrée en 1987 et 1988. Les différences de croissance entre les traitements ombrés et le témoin étaient significatives dès le premier prélèvement, soit $9 \mathrm{j}$ après la pose des filets en 1987 (prélèvement du 4 juin 1987 ) et 5 jours en 1988 (prélèvement du 1er juin 1988). Lors de l'enlèvement des filets, la biomasse aérienne par plante était respectivement de $16,3 \%$ (ombrage II) et $10,8 \%$ (ombrage III) de celle des plantes non ombrées en 1987 et de $89,4 \%$ (ombrage I), 30,0\% (ombrage II) et $18,8 \%$ (ombrage III) en 1988. Dans les traitements ombrés, il y a eu également un retard d'apparition des feuilles. Lors de l'enlèvement

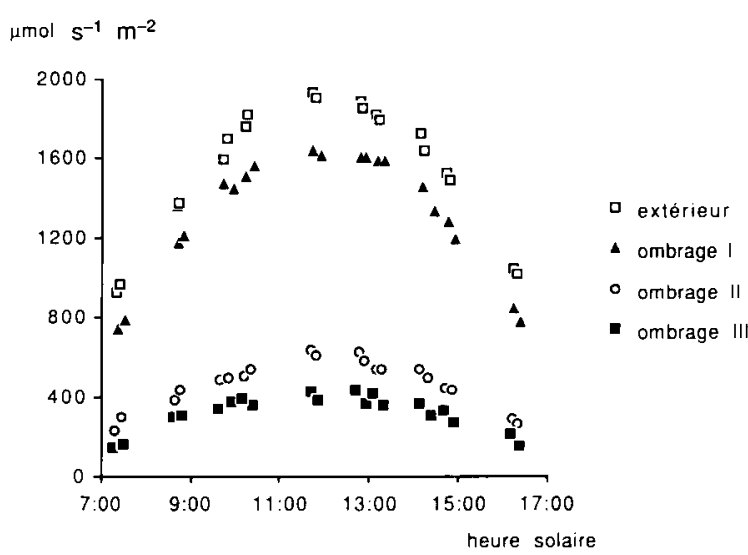

Fig 2. Rayonnement mesuré au-dessus du couvert en fonction de l'heure dans les différents traitements (heure solaire); mesures du 24 juin 1988. 

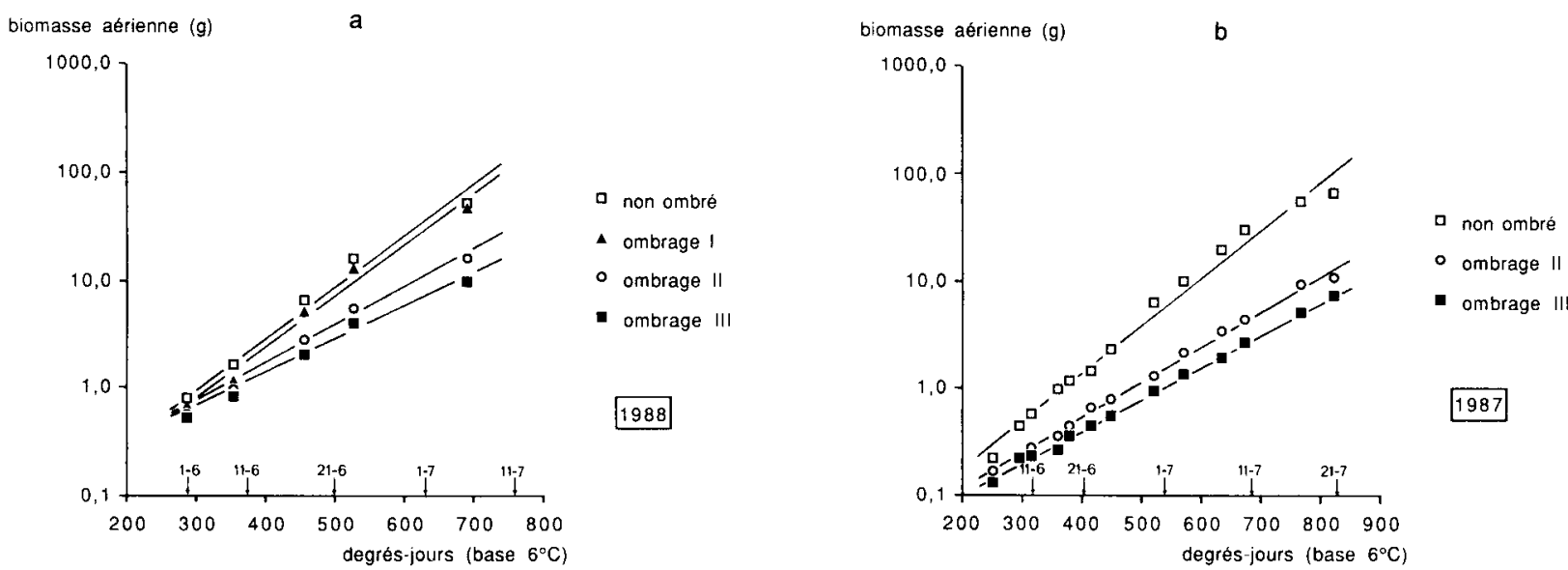

Fig 3. Evolution de la biomasse aérienne par plante en fonction des sommes de degrés-jours en base $6^{\circ} \mathrm{C}$ après semis. a. $1987 ; \boldsymbol{b}$. 1988.

des filets, le nombre de feuilles visibles était respectivement de $16,4,13,2$ et 12,4 dans les 3 traitements en 1987 et de $15,6,15,0,12,8$ et 11,8 en 1988. On observe en coordonnées logarithmiques une relation peu différente entre traitements entre le nombre de feuilles visibles ( $n f v$ ) et la biomasse aérienne par plante $(\mathrm{ms})$ ( $n \mathrm{fv}=$ $\left.4,295 \log _{10}(\mathrm{~ms})+7,826 ; r^{2}=0,960\right)$.

\section{Développement du système racinaire}

\section{Rythme d'apparition des racines adventives}

La figure $4 a, b$, représentent l'évolution du nombre d'entre-nœuds portant des racines et l'évolution du nombre total de racines adventives émises par plante pendant la période ombrée en 1987. Dans le traitement non ombré, on observe un arrêt de l'émission racinaire vers 300 degrés-

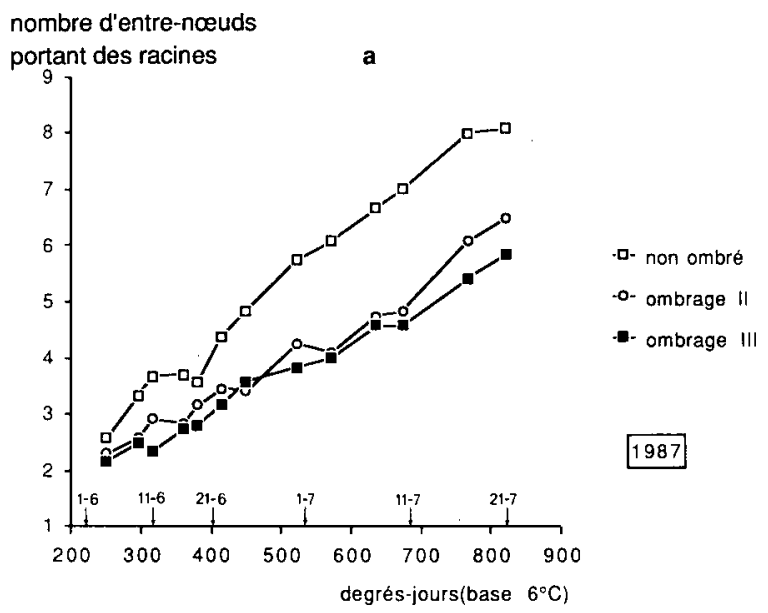

jours après semis, ce qui coïncide au début de la translocation des produits de la photosynthèse vers les racines (Deleens et al, 1984; Derieux et al, 1989). Ces auteurs ont observé pendant cette période un arrêt de la croissance racinaire que l'on retrouve donc également au niveau du développement. La comparaison des différents traitements montre un retard de l'émission racinaire sous les ombrages. Le même résultat a été obtenu en 1988 (données non présentées). II existe une relation linéaire entre le nombre d'entrenœuds portant des racines et le nombre de feuilles visibles (fig 5). Cette relation a déjà été signalée par d'autres auteurs (Picard et al, 1985; Jordan et al, 1988). Une analyse de covariance montre qu'elle est indépendante du traitement en $1988(F=2,78)$ mais pas en $1987(F=6,56)$ où pour un même stade foliaire, le nombre de niveaux de racines est un peu plus faible dans les traitements ombrés. Les relations obtenues sont cependant très proches (fig 5). L'application des

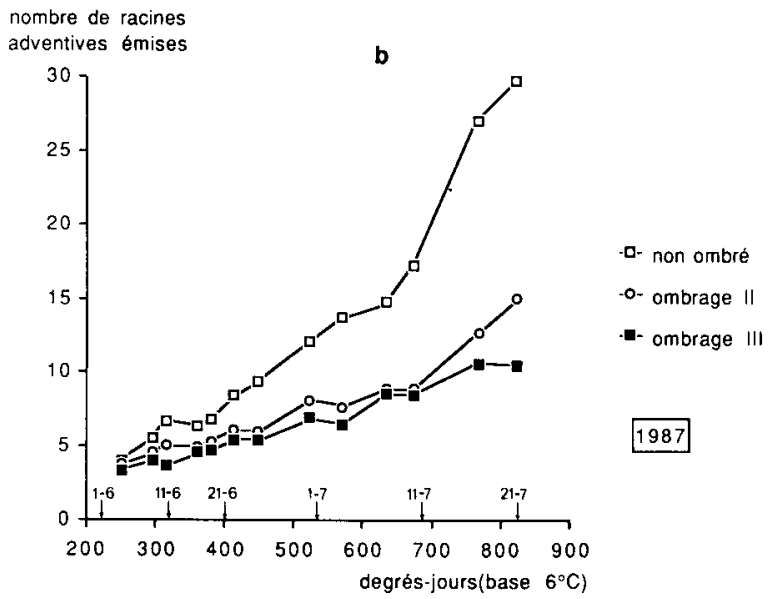

Fig 4. Evolution du nombre d'entre-nœuds portant des racines a et du nombre total de racines adventives par plante $\mathbf{b}$ en fonction des sommes de température après semis en 1987. 
nombre d'entre-nceuds

portant des racines

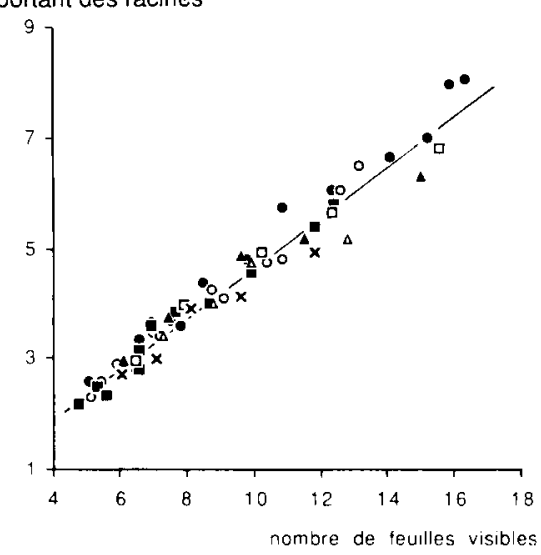

Fig 5. Relation entre le nombre d'entre-nœuds ayant émis des racines (ne) et le nombre de feuilles visibles ( $n f v$ ). $n e=0,459 ; n f v+0,105 ; R^{2}=0,962$.

ombrages a donc très peu modifié la synchronie entre l'apparition des feuilles et l'émission des racines sur les entre-nœuds successifs.

Dans la figure 6, apparaît en coordonnées logarithmiques le nombre total de racines adventives en fonction de la biomasse aérienne par plante, pendant la période ombrée pour tous les traitements. La relation obtenue est très peu différente entre traitements. Pendant l'émission sur les entre-nœuds $E_{2}$ à $E_{5}$, la relation nombre de racines émises - $\log _{10}(m s)$ est linéaire. Une analyse de covariance montre qu'elle est indépendante du traitement et de l'année.

\section{Nombre de racines adventives par entre-nœud}

La figure $7 a, b$ et le tableau I représentent les nombres de racines par entre-nœud à la récolte dans les différents traitements et les corrélations

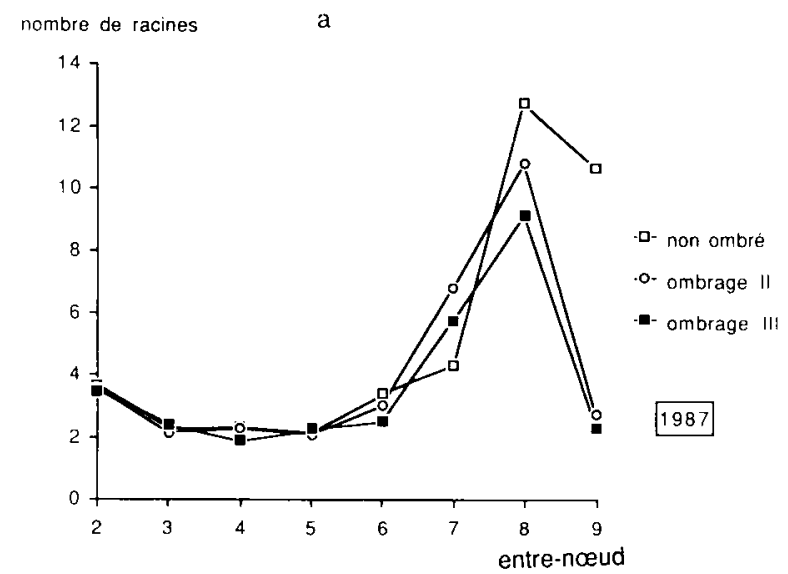

Fig 7. Nombre de racines par entre-nœud à la récolte. a. 1987; b. 1988

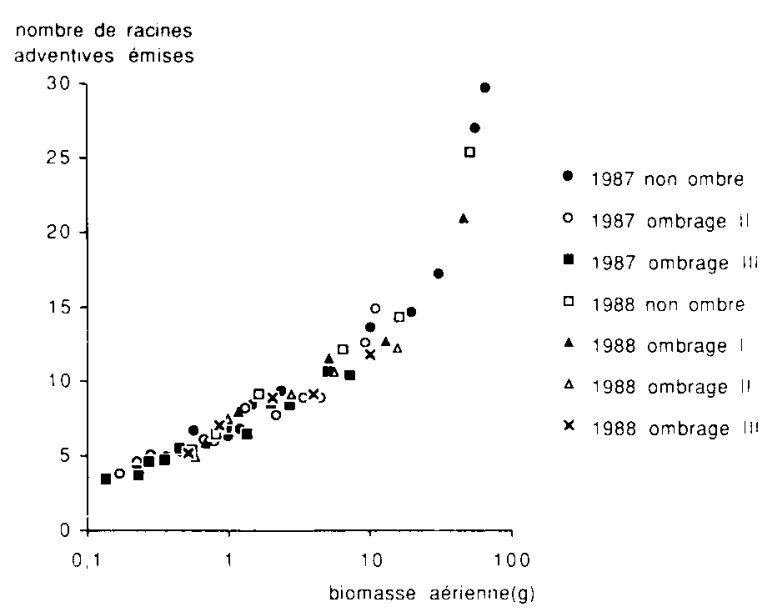

Fig 6. Relation entre le nombre de racines adventives émises et la biomasse aérienne des plantes.

entre entre-nœuds successifs. Dans le tableau II, sont indiqués les entre-nœuds sur lesquels l'émission des racines commençait ou était en cours au moment de l'enlèvement des filets. On a considéré que l'émission des racines était terminée sur un entre-nœud si tous les pieds prélevés portaient déjà des racines sur l'entre-nœud suivant ou bien si le nombre de racines sur cet entre-nœud n'était pas significativement différent du nombre de racines final compté à la récolte. Pour les entre-nœuds sur lesquels l'émission des racines était en cours lors de l'enlèvement des filets, on a indiqué le nombre de racines déjà émises à cette date et le nombre de racines final à la récolte (tableau II).

Quels que soient l'année et le traitement, les nombres de racines sur les entre-nœuds $E_{3}$ et $E_{4}$ d'une part, $E_{4}$ et $E_{5}$ d'autre part, sont corrélés négativement (tableau I). En 1987 et en 1988, l'émission des racines sur les entre-nœuds $E_{2}$ à

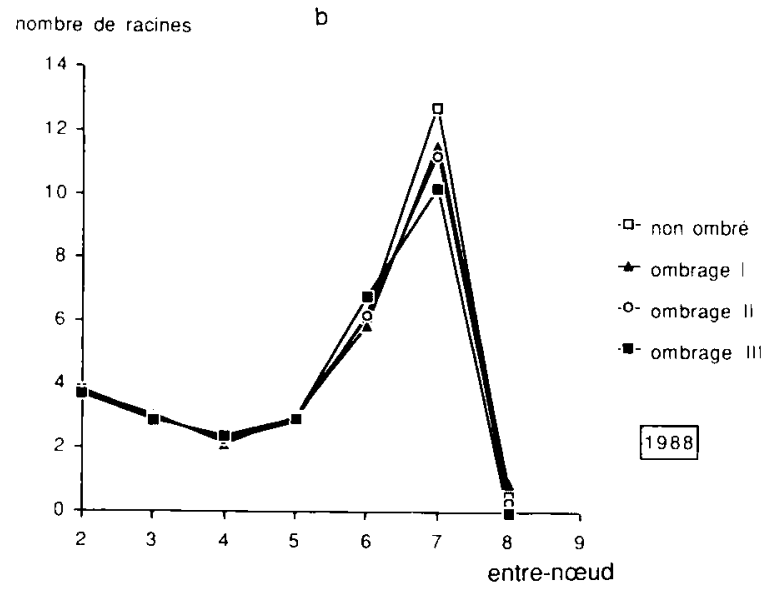


Tableau I. Coefficients de corrélation entre nombres de racines sur 2 entre-nœuds successifs.

\begin{tabular}{lcccccc}
\hline Traitement & \multicolumn{5}{c}{ Entre-nœuds } \\
& $E_{2} / E_{3}$ & $E_{3} / E_{4}$ & $E_{4} / E_{5}$ & $E_{5} / E_{6}$ & $E_{6} / E_{7}$ & $E_{7} / E_{8}$ \\
\hline Témoin non ombré 1987 & 0,048 & $-0,276$ & $-0,416$ & $-0,156$ & $-0,053$ & 0,261 \\
Ombrage II 1987 & $-0,181$ & $-0,441$ & $-0,685$ & $-0,186$ & 0,105 & 0,661 \\
Ombrage III 1987 & $-0,304$ & $-0,451$ & $-0,712$ & $-0,259$ & 0,061 & 0,471 \\
Témoin non ombré 1988 & 0,157 & $-0,520$ & $-0,543$ & 0,014 & 0,260 & $-0,294$ \\
Ombrage I 1988 & 0,113 & $-0,370$ & $-0,409$ & 0,103 & $-0,244$ & $-0,068$ \\
Ombrage II 1988 & 0,162 & $-0,418$ & $-0,402$ & $-0,090$ & $-0,080$ & 0,040 \\
Ombrage III 1988 & 0,121 & $-0,300$ & $-0,415$ & $-0,178$ & $-0,313$ & - \\
\hline
\end{tabular}

$E_{5}$ a eu lieu entièrement pendant la période ombrée (tableau II). II n'apparaît pas de différence entre les traitements ombrés et non ombrés quant au nombre de racines sur ces entrenœuds (fig 7). En 1987, le nombre total de racines sur $E_{2}-E_{5}$ n'est pas significativement différent entre traitements (respectivement 10,37, 10,19 et 10,13 racines pour le traitement non ombré et les ombrages II et III). En 1988, le nombre total de racines sur $E_{2}-E_{5}$ est significativement plus faible au seuil $5 \%$ sous l'ombrage II mais pas sous l'ombrage III (respectivement $12,05,11,85,11,75$ et 11,88 racines pour le traitement non ombré et les ombrages I, II et III). En
1987, l'émission des racines sur $E_{6}$ a eu lieu en partie pendant la période ombrée (tableau II). Le nombre de racines final sur cet entre-nœud est significativement plus faible pour les plantes ombrées $(3,03$ et 2,54 racines respectivement sous les ombrages II et III) que pour les plantes non ombrées (3,41 racines). Sur l'entre-nœud 7 , pour lequel l'émission a eu lieu entièrement (ombrage III) ou en grande partie (ombrage II) après l'enlèvement des filets, on observe au contraire plus de racines dans les traitements ayant été préalablement ombrés. L'ordre des traitements s'inverse à nouveau pour $E_{8}$ et $E_{9}$. En 1988, l'émission sur $E_{6}$ a eu lieu pendant la période ombrée

Tableau II. Nombre de racines à la récolte et lors de l'enlèvement des ombrages pour les entre-nœuds sur lesquels l'émission racinaire était en cours à cette date.

Entre-nœud

Ombrage II 1987

Ombrage III 1987

Ombrage I 1988

Ombrage II 1988

Ombrage III 1988

\section{$E_{6}$}

$E_{7}$

$E_{6}$

$E_{7}$

$E_{6}$

$E_{6}$
Nombre de racines

lors de l'enlèvement à la récolte
des filets

2,17

3,03

2,08

6,83

1,08

2,54

3,04

11,56

0,75

6,18

0,00

6,77 
uniquement sous l'ombrage I. Le nombre de racines sur cet entre-nœud est plus faible sur les plantes ayant subi cet ombrage $(5,86)$ que sur les plantes non ombrées $(6,14)$ mais la différence n'est pas significative. Dans les ombrages $\|$ et III, l'émission sur $E_{6}$ s'est faite après l'enlèvement des ombrages. On observe, comme pour $E_{7}$ en 1987 plus de racines dans les traitements ayant été ombrés au préalable. L'ordre des traitements s'inverse à nouveau pour $E_{7}$ et $E_{8}$.

\section{DISCUSSION ET CONCLUSIONS}

L'utilisation d'ombrages pour notre expérience a permis de limiter fortement le rayonnement disponible, et donc la croissance des plantes en début de cycle. Les relevés de température effectués montrent qu'il y a eu concomitamment réduction de la température moyenne du sol sous les traitements ombrés. Cet effet n'était pas souhaité mais il est difficilement évitable. On peut penser cependant que les écarts mesurés sont supérieurs aux écarts subis au niveau des apex des plantes. La température au niveau de l'apex est a priori intermédiaire entre la température du sol mesurée à $5 \mathrm{~cm}$ de profondeur et la température de l'air qui est très peu différente entre traitements. Par ailleurs, du fait d'un retard involontaire dans la pose des thermomètres, les mesures de température du sol ont été effectuées uniquement entre le 15 juin et le 6 juillet 1988, c'est-à-dire pendant une période de fortes températures. En 1987, il est probable que les écarts entre traitements ont été plus faibles, du fait des températures basses au printemps. Ces écarts de température du sol peuvent cependant expliquer en partie le retard de développement constaté dans les traitements ombrés.

L'application des ombrages a entraîné un retard dans l'apparition à la fois des feuilles et des racines. La synchronie entre les émissions foliaires et racinaires a été très peu modifiée. Cela montre la stabilité de la relation mise en évidence par Picard et al (1985) et son intérêt dans une optique de modélisation.

Sur les entre-nœuds $E_{2}-E_{5}$, le nombre de racines est identique dans les traitements ombrés et non ombrés, bien que l'émission racinaire ait eu lieu pendant la période ombrée. Sur ces entre-nœuds, le nombre de racines émises ne serait donc pas régulé en fonction de l'alimentation carbonée de la plante. La moindre croissance des plantes sous les ombrages a entraîné un retard de l'émission racinaire mais sans modi- fication du nombre de racines finalement émises. La relation biomasse aérienne-nombre de racines pendant l'émission sur $E_{2}-E_{5}$ est identique, quel que soit le traitement. Sur $\mathrm{E}_{6}$ par contre, le nombre de racines est plus faible dans les traitements ombrés quand l'émission racinaire sur cet entre-nœud a eu lieu pendant la période d'ombrage. Ces observations sont à rapprocher des résultats obtenus par Picard et al (1985), Hoppe et al (1986) et Girardin et al (1987). D'après ces travaux, la variabilité du nombre de racines est faible sur $E_{2}-E_{5}$ et augmente à partir de $E_{6}$. Sur $E_{6}-E_{9}$, le nombre de racines émises est inférieur au nombre d'ébauches racinaires présentes. D'après l'ensemble de ces résultats, le nombre de racines sur ces entrenœuds pourrait donc être régulé en fonction de la quantité disponible d'assimilats. L'augmentation observée du nombre de racines sur les entre-nœuds dont l'émission racinaire a eu lieu juste après l'enlèvement des ombrages pourrait être dû à l'accroissement brutal ainsi provoqué de la photosynthèse. La différence observée entre les 2 années (respectivement 19,4 et 31,1 racines sur $E_{6}-E_{9}$ dans les traitements non ombrés en 1987 et 1988) confirme la très grande variabilité du nombre de racines sur ces entre-nœuds. Dans une optique de modélisation de l'architecture racinaire tenant compte des relations avec les parties aériennes, ces observations montrent la nécessité d'étudier les relations entre la disponibilité en assimilats et l'émission racinaire sur ces entre-nœuds.

Sur $E_{2}-E_{5}$, le travail réalisé n'a pas permis d'identifier l'origine de la variabilité du nombre de racines émises. Bien qu'elle soit faible, la variabilité sur ces entre-nœuds est importante à considérer, car ces racines sont les seules qui colonisent l'interrang, d'où l'importance de leur nombre. Les racines des entre-nœuds supérieurs deviennent très rapidement verticales et colonisent donc seulement la partie du profil située sous le rang (Tardieu et Pellerin, 1990). Compte tenu des résultats obtenus par Girardin et al (1987), l'origine de la variabilité sur les entre-nœuds $E_{2}-E_{5}$ se situerait au niveau du nombre d'ébauches différenciées, donc relativement tôt au cours du cycle. On peut observer que le nombre total de racines sur ces entrenœuds a été significativement plus faible en 1987 (10,37 racines) qu'en 1988 (12,05 racines). Une hypothèse possible pourrait être que cet écart est dû aux températures plus faibles en 1987 en début de cycle. Pour tester cette hypothèse, il serait nécessaire d'étudier l'effet de la 
température sur le nombre d'ébauches racinaires différenciées sur ces entre-nœuds.

\section{RÉFÉRENCES}

Aresta RB, Fukai S (1984) Effects of solar radiation on growth of cassava (Manihot esculenta Crantz). II. Fibrous root length. Field Crops Res 9, 361-371

Bourdu R, Gregory N (1983) Etude comparée du début de la croissance chez divers génotypes de mais. Agronomie 3, 761-770

Del Castillo D, Acock B, Reddy VR, Acock MC (1989) Elongation and branching of roots on soybean plants in a carbon dioxyde-enriched aerial environment. Agron J 81, 692-695

Deleens E, Grégory N, Bourdu R (1984) Transition between seed reserve use and photosynthetic supply during development of maize seedlings. Plant Sci Lett 37, 34-39

Derieux M, Bourdu R, Duburcq JB, Boizard H (1989) La crise de croissance de la plantule de maïs à basse température. Agronomie 9, 207-212

Diggle AJ (1988) ROOTMAP. A model in threedimensional coordinates of the growth and structure of fibrous root system. Plant Soil 105, 169-178

Girardin P, Jordan MO, Picard D, Trendel R (1986) Harmonisation des notations concernant la description morphologique d'un pied de maïs (Zea mays L). Agronomie 6, 873-875

Girardin P, Morel-Fourrier B, Jordan MO, Millet B (1987) Développement des racines adventives chez le maïs. Agronomie 7, 353-360
Hoppe DC, Mc Cully ME, Wenzel CL (1986) The nodal roots of Zea: their development in relation to structural features of the stem. Can J Bot 64, 2524-2537

Jordan MO, Girardin P, Varlet-Grancher C, Picard D, Trendel R (1988) Rythme d'apparition des racines primaires du maîs (Zea mays L). III. Variations observées au champ. Agronomie 8, 37-46

Pagès L, Ariès F (1988) Sarah : modèle de simulation de la croissance, du développement et de l'architecture des systèmes racinaires. Agronomie 8, 889896

Pagès L, Jordan MO, Picard D (1989) A simulation model of the three-dimensional architecture of the maize root system. Plant Soil 119, 147-154

Picard D, Jordan MO, Trendel R (1985) Rythme d'apparition des racines primaires du maïs (Zea mays L). I. Etude détaillée pour une variété en un lieu donné. Agronomie 5, 667-676

Porter JR, Klepper B, Belford RK (1986) A model (WHTROOT) which synchronizes root growth and development with shoot development for winter wheat. Plant Soil 92, 133-145

Tardieu F, Pellerin S (1990) Trajectory of the nodal roots of maize in fields with low mechanical constraints. Plant Soil 124, 39-45

Vincent CD, Grégory PJ (1989a) Effects of temperature on the development and growth of winter wheat roots. I. Controlled glasshouse studies of temperature, nitrogen and irradiance. Plant Soil $119,87-97$

Vincent CD, Grégory PJ (1989b) Effects of temperature on the development and growth of winter wheat roots. II. Field studies of temperature, nitrogen and irradiance. Plant Soil 119, 99-110 